\title{
STRATIFICATIONS OF POLYNOMIAL SPACES
}

\author{
L. BIRBRAIR
}

\begin{abstract}
In the paper we construct some stratifications of the space of monic polynomials in real and complex cases. These stratifications depend on properties of roots of the polynomials on some given semialgebraic subset of $\mathbb{R}$ or $\mathbb{C}$. We prove differential triviality of these stratifications. In the real case the proof is based on properties of the action of the group of interval exchange transformations on the set of all monic polynomials of some given degree. Finally we compare stratifications corresponding to different semialgebraic subsets.
\end{abstract}

\section{Introduction}

In Theory of Singularities many authors use the following conception: local (or global) stability follows from transversality to some stratification of some space of polynomial (analytic or smooth) maps (see, for example, [AVG], [dPW]). Here we present some stratifications of spaces of monic polynomials on one variable connected to problems of stability of singularities of projection and singularities near boundary points of hypersurfaces.

In the proof of Tarski-Seidenberg Theorem (see, for example, [BR]) appears the following partition $\left\{Q_{k}\right\}$ of the space of monic polynomials: $f \in Q_{k}$ if $f$ has exactly $k$ roots. In fact the main step of the proof of Tarski-Seidenberg Theorem is to prove that $Q_{k}$ are semialgebraic subsets. The partition $\left\{Q_{k}\right\}$ is not a stratification. In [GWPL] it is mentioned that if we consider not just a number of roots but multiplicities of these roots we obtain a Whitney regular stratification of the space of monic polynomials.

In $[\mathbf{G}]$ it is proved that if we fix multiplicities of the eigenvalues of $n \times n$ matrices and the structures of their Jordan blocks (so-called Segre symbol) we obtain a stratification of the space of $n \times n$ matrices. C. G. Gibson also proved that this stratification is Whitney regular. 
In the present paper we consider some generalizations of the stratification considered in $[\mathbf{G W P L}]$. Namely, let $N$ be some closed semialgebraic subset of $\mathbb{C}$. Thus we have the following decomposition $N=\operatorname{Int}(N) \cup S_{1} \cup S_{2} \cup \operatorname{Sing}(\partial N)$, where $\operatorname{Int}(N)$ is the set of internal points of $N ; S_{1} \subset \partial N$ is a subset of the border $\partial N$ contains only $C^{\rho}$-smooth points which have the internal points of $N$ in every small neighbourhood; $S_{2}$ is a complement to $S_{1}$ in the set of $C^{\rho}$-smooth points of $\partial N$; as usual $\operatorname{Sing}(\partial N)$ is a subset of $C^{\rho}$-singular points of $\partial N$. We define a multiplicity symbol (see sections 2.1 and 3.1 ) which is determined by multiplicities of the roots of given polynomial on $\operatorname{Int}(N), S_{1}$, $S_{2}$ and $\operatorname{Sing}(N)$.

The main result of the paper is the theorem that all stratifications given by these multiplicity symbols are differentially trivial and thus Whitney regular (Theorems 2.4 and 3.1). Observe that the stratifications under considerations are different and depend on $N$ and $\rho$. In the case $N=\mathbb{C}$ we obtain the stratification considered in $[\mathbf{G W P L}]$.

The paper has the following structure. Part 2 is devoted to stratifications of the space of real monic polynomials. Since any closed semialgebraic subset of $\mathbb{R}$ is or $\mathbb{R}$ itself, or a finite union of closed segments, points and closed halflines we begin our consideration from the case of finite segment. The case of the union of segments and halflines can be treated in the same way. We define a multiplicity symbol for polynomials. This multiplicity symbol is connected to some fixed segment $[b, c]$ and characterizes the number of roots a polynomial has on this segment and their multiplicities. For each multiplicity symbol we define a stratum (the set of all polynomials with the same multiplicity symbol). We prove that the stratification by multiplicity symbols gives us a semialgebraic stratification. It is known [BCR] that each semialgebraic stratification can be finitely subdivided to obtain a semialgebraic stratification satisfying " $a$ " and " $b$ " axioms of Whitney. The interesting property of the stratification by multiplicity symbol is the following: it is not necessary to subdivide it because it is Whitney regular itself. Paragraphs 2.3 and 2.4 are devoted to proving this fact. Section 2.3 is devoted to Interval Exchange Transformations [Ke]. Interval Exchange Transformation is a very popular object in ergodic theory. Here we use it in real algebraic geometry. We prove that all the strata defined in 2.1 are invariant by an action of group of interval exchange transformations. The action of any nontrivial interval exchange transformation on the space of polynomials is not continuous, but if the roots of some polynomial are well situated the action of a given interval exchange transformation is a difeomorphism on a neighbourhood of the polynomial. This important property of this group action helps us a lot of prove in section 2.4 that the considering 
stratifications satisfy the boundary axiom. In fact the boundary axiom can be obtained as a corollary of Whitney regularity, but the semiorder relation itself has a nice combinatorial and geometrical nature, which gives us a more detailed description of the stratifications.

Part 3 is connected to the complex case, where $N$ is a closed semialgebraic subset of $\mathbb{C}$. To prove a differential triviality we consider socalled disk exchange transformations, which correspond to interval exchange transformations in real case. These transformations have not such nice properties as interval exchange transformations (they do not form a group), but they are useful to prove a differential triviality of the stratifications under consideration.

Part 4 is devoted to the comparing of different stratifications. In section 4.1 we compare real and complex stratifications for the same $N$. In section 4.2 we prove that two stratifications $\left\{P_{n, \mu\left(N_{1}\right)}\right\}$ and $\left\{P_{n, \mu\left(N_{2}\right)}\right\}$ (see the notations of part 3 ) are $C^{\rho}$-equivalent if the corresponding subsets $N_{1}$ and $N_{2}$ are $C^{\rho}$-equivalent.

Finally I'd like to mention that these stratifications are rather natural. If we consider a partition $\left\{Q_{k}(N)\right\}$ (the similar as in [BR]) given by the number of roots of some polynomial in $N$ and apply the algorithm defined in $[\mathbf{B C R}]$ to obtain a Whitney regular stratification we will obtain exactly the stratification by multiplicity symbol.

\section{Stratifications of real monic polynomials}

\subsection{Multiplicity symbol.}

Let $P_{n}$ be a space of all monic polynomials of degree $n$ with real coefficients. $P_{n}$ can be identified to the space $\mathbb{R}^{n}$ in the standard way:

$$
f=u^{n}+a_{n-1} u^{n-1}+\cdots+a_{1} u+a_{0} \in P_{n} \leftrightarrow\left(a_{0}, a_{1}, \ldots, a_{n-1}\right) \in \mathbb{R}^{n} .
$$

Consider some (closed) segment $[b, c]$ in $\mathbb{R}$. Let's define the symbol $\mu=\left\{\mu_{b}, \mu_{1}, \ldots, \mu_{r}, \mu_{c}\right\}$ corresponding to $[b, c]$ such that all numbers $\mu_{b}$, $\mu_{c}, \mu_{i}(1 \leq i \leq r)$ satisfy the following conditions:

1. $0 \leq r \leq n$.

(Note that we consider also $r=0$; in this case $\mu=\left\{\mu_{b}, \mu_{c}\right\}$ ).

2. $\mu_{b}, \mu_{c} \in \mathbb{N} \cup\{0\} ; \mu_{i} \in \mathbb{N}$.

( $\mathbb{N}$ is the set of natural numbers).

3. $\sum_{i=1}^{r} \mu_{i}+\mu_{b}+\mu_{c} \leq n$.

4. $1 \leq \mu_{1} \leq \cdots \leq \mu_{r}$. 
Let $\mathcal{M}$ be the set of all these symbols $\mu$.

Denote by $P_{n, \mu}$ the set of polynomials $f \in P_{n}$ such that

1. $f$ has roots of multiplicities $\mu_{b}$ and $\mu_{c}$ at the points $b$ and $c$ correspondently.

(Note that $\mu_{b}=0\left(\right.$ or $\left.\mu_{c}=0\right)$ means that $f(b) \neq 0($ or $f(c) \neq 0)$ ).

2. $f$ has exactly $r$ (different) roots $v_{1} \neq v_{2} \neq \cdots \neq v_{r}$ of multiplicities $\mu_{1}, \mu_{2}, \ldots, \mu_{r}$ (correspondently) in the open interval $(b, c)$.

In other words $P_{n, \mu}$ is defined by the intersection of the zero divisor of $f$ with the segment $[b, c]$. Note that $P_{n, \mu}$ depends on the segment $[b, c]$.

Theorem 2.1. The collection $\left\{P_{n, \mu}\right\}_{\mu \in \mathcal{M}}$ is a semialgebraic stratification of $P_{n}$.

Proof: It is clear that $\bigcup_{\mu \in \mathcal{M}} P_{n, \mu}=P_{n}$ because each polynomial belongs to some $P_{n, \mu}$ (for some $\mu$ ). To prove that all $P_{n, \mu}$ are smooth submanifolds and semialgebraic sets we need the following.

Lemma 2.1. Let $\mu=\{0, \overbrace{1,1, \ldots, 1}^{r}, 0\}$ ( $r$ can be equal to 0$)$. Then $P_{n, \mu}$ is an open semialgebraic set in $P_{n}$.

Proof of Lemma 2.1: Let us prove that $P_{n, \mu}$ is semialgebraic. Each polynomial $f \in P_{n, \mu}$ can be presented in the following form:

(1) $f=\left(u-v_{1}\right) \cdot \ldots \cdot\left(u-v_{r}\right)\left(u-\ell_{1}\right) \cdot \ldots \cdot\left(u-\ell_{s}\right)$

$$
\cdot\left(u^{2}+p_{1} u+q_{1}\right) \cdot \ldots \cdot\left(u^{2}+p_{t} u+q_{t}\right)
$$

for some $s, t \in \mathbb{N} \cup\{0\}$ uniquely determined by $f$ such that $r+s+2 t=n$.

For given pair $(s, t)$ the set $P_{n, \mu}$ defines a semialgebraic set $U(s, t)$ in the space $\mathbb{R}^{n}$ of coefficients $\left(v_{1}, \ldots, v_{r}, \ell_{1}, \ldots, \ell_{s}, p_{1}, q_{1}, \ldots, p_{t}, q_{t}\right)$ by the following inequalities:

$$
\begin{cases}b<v_{i}<c, & i=1,2, \ldots, r\left(\text { it means: } v_{i} \in(b, c)\right) \\ v_{i} \neq v_{j} \text { for } i \neq j ; & i, j=1,2, \ldots, r \\ \ell_{i}<b \text { or } \ell_{i}>c, & \left.i=1,2, \ldots, s \text { (it means: } \ell_{i} \notin[b, c]\right) \\ p_{j}^{2}-4 q_{j}<0, & j=1,2, \ldots, t .\end{cases}
$$


Let $Q_{s, t}: \mathbb{R}^{n} \rightarrow P_{n}$ be a map obtained by the opening brackets in (1). (Remined that $P_{n}$ is identified to $\mathbb{R}^{n}$ ). We get

$$
P_{n, \mu}=\bigcup_{\substack{s, t \\ s+r+2 t=n}} Q_{s, t}(U(s, t)) .
$$

Since $Q_{s, t}$ is an algebraic map we obtain (by Tarski-Seidenberg Theorem) that $P_{n, \mu}$ is a semialgebraic set.

Let's prove that $P_{n, \mu}$ is an open set. By the definition of $P_{n, \mu}$ the graph $f$ considered in the subset $(b, c) \times \mathbb{R}$ intersects the "zero section" $(b, c) \times\{0\}$ transversally. Hence there exists a neighbourhood of $f$ in $P_{n}$ satisfies the same property. It means that $P_{n, \mu}$ is an open subset. Lemma 2.1 is proved.

Lemma 2.2. $P_{n, \mu}$ is a smooth submanifold of $P_{n}$ and $\operatorname{codim} P_{n, \mu}=$ $\mu_{b}+\mu_{c}+\sum_{i=1}^{r}\left(\mu_{i}-1\right)$.

We prove this lemma in several steps.

Claim. Let $\mu$ satisfies the following condition:

$$
\sum_{i=1}^{r} \mu_{i}+\mu_{b}+\mu_{c}=n .
$$

Then $P_{n, \mu}$ is a smooth submanifold of $P_{n}$.

Proof: Consider a space $\mathbb{R}^{r}$ and a subset $V_{\mu} \subset \mathbb{R}^{r}$ defined in the following way: $V_{\mu}=\left\{\left(v_{1}, \ldots, v_{r}\right) \in \mathbb{R}^{r}\right.$ such that

$$
\begin{cases}v_{i} \in(b, c), & i=1, \ldots, r \\ v_{i} \neq v_{j}, & \text { if } i \neq j \\ v_{i}<v_{j}, & \text { if } \left.\mu_{i}=\mu_{j} \text { and } i<j\right\} .\end{cases}
$$

Let $F_{\mu}: V_{\mu} \rightarrow P_{n}$ be the following map

$$
\text { (3) } \begin{array}{r}
F_{\mu}\left(v_{1}, \ldots, v_{r}\right)=\left(u-v_{1}\right)^{\mu_{1}} \cdot\left(u-v_{2}\right)^{\mu_{2}} \cdot \ldots \cdot\left(u-v_{r}\right)^{\mu_{r}} \\
\cdot(u-b)^{\mu_{b}} \cdot(u-c)^{\mu_{c}} .
\end{array}
$$

$F_{\mu}$ is a one-to-one map because each polynomial is uniquely defined by it's roots. 
$F_{\mu}\left(V_{\mu}\right)=P_{n, \mu}$ because for each polynomial $f \in P_{n, \mu}$ we have the presentation (3).

Now we have to prove that $F_{\mu}$ is an immersion. We do it using the induction by $n$.

If $n=1$ we have:

$P_{1,\{0,1\}}$ and $P_{1,\{1,0\}}$ are one points each,

$P_{1,\{0,1,0\}}$ is an open interval $\left(V_{\{0,1,0\}}=\{v \in(b, c)\}, F_{\{0,1,0\}}(v)=u-v\right)$.

Now suppose that $F_{\mu}$ is an immersion for each $n<n_{0}$. Let's prove it for $n_{0}$.

Suppose that $r \neq 0$. For each polynomial $f \in P_{n_{0}, \mu}$ we have the following presentation:

$$
f=\left(u-v_{r}\right)^{\mu_{r}} \cdot h
$$

where $v_{r}$ is a root of $f$ belonging to $(b, c)$ and $h \in P_{n_{0}-\mu_{r}, \nu}, \nu=$ $\left\{\mu_{b}, \mu_{1}, \ldots, \mu_{r-1}, \mu_{c}\right\}$.

Let $G_{\mu_{r}}:(b, c) \times P_{n_{0}-\mu_{r}} \rightarrow P_{n_{0}}$ be the following map:

$$
G_{\mu_{r}}\left(v_{r}, g\right)=\left(u-v_{r}\right)^{\mu_{r}} \cdot g
$$

where $g \in P_{n_{0}-\mu_{r}}$.

We will prove that $G_{\mu_{r}}$ is a local immersion in a neighbourhood of a point $\left(v_{r}, g\right)$ such that $v_{r}$ is not a root of $g$. Thus we can consider the map $F_{\mu}$ as the following:

$$
F_{\mu}\left(v_{1}, \ldots, v_{r}\right)=\left.G_{\mu_{r}}\left(v_{r}, F_{\nu}\left(v_{1}, \ldots, v_{r-1}\right)\right)\right|_{V_{\mu}}
$$

and obtain that $F_{\mu}$ is an immersion $\left(F_{\nu}\right.$ is an immersion by the induction hypothesis).

Let $U_{v_{r}}$ and $U_{g}$ be neighbourhoods of $v_{r}$ and $g$ such that for each $v \in U_{v_{r}}$ and for each $\tilde{g} \in U_{g} v$ is not a root of $\tilde{g}$. In local coordinates (putting $\tilde{u}=u-v_{r}$ ) we obtain

$$
\tilde{g}(\tilde{u})=\tilde{u}^{n_{0}-\mu_{r}}+\tilde{g}_{n_{0}-\mu_{r}-1} \tilde{u}^{n_{0}-\mu_{r}-1}+\cdots+\tilde{g}_{1} \tilde{u}+\tilde{g}_{0},
$$

where $\tilde{g}_{0} \neq 0$ because $v_{r}$ is not a root of $\tilde{g}$.

Putting this presentation (6) to (5) we obtain

$$
G_{\mu_{r}}(v, \tilde{g})=\tilde{u}^{n_{0}}+q_{n_{0}-1} \tilde{u}^{n_{0}-1}+\cdots+q_{1} \tilde{u}+q_{0},
$$


where

$$
\left\{\begin{aligned}
q_{n_{0}-1} & =\tilde{g}_{n_{0}-\mu_{r}-1}+\mu_{r} \cdot w \\
q_{n_{0}-2} & =\tilde{g}_{n_{0}-\mu_{r}-2}+\mu_{r} \cdot w \cdot \tilde{g}_{n_{0}-\mu_{r}-1}+o(w) \\
& \ldots \ldots \ldots \ldots \ldots \ldots \ldots \ldots \ldots \ldots \ldots \ldots \ldots \ldots \ldots \ldots \\
q_{\mu_{r}} & =\tilde{g}_{0}+\mu_{r} \cdot w \cdot \tilde{g}_{1}+o(w) \\
q_{\mu_{r}-1} & =\mu_{r} \cdot w \cdot \tilde{g}_{0}+o(w) \\
q_{i} & =o(w) \text { for } i=0, \ldots, \mu_{r}-2 .
\end{aligned}\right.
$$

(We use the formula: $(\tilde{u}+w)^{\mu_{r}}=\tilde{u}^{\mu_{r}}+\mu_{r} \tilde{u}^{\mu_{r}-1} w+o(w) ; w=v_{r}-v$.)

From (7) we can see that the Jacobian matrix of $G_{\mu_{r}}$ has an above triangular structure. Since $\tilde{g}_{0} \neq 0$ it has a maximal rank. Thus the map $G_{\mu_{r}}$ is a local immersion in a neighbourhood of $\left(v_{r}, g\right)$.

If $r=0$ symbol $\mu$ has the following form: $\left\{\mu_{b}, \mu_{c}\right\}$ and $\mu_{b}+\mu_{c}=n_{0}$. Hence $P_{n_{0}, \mu}$ contains only one point $f=(u-b)^{\mu_{b}} \cdot(u-c)^{\mu_{c}}$.

So, we proved that $P_{n, \mu}$ is an immersed submanifold. To prove that $P_{n, \mu}$ is a submanifold we'll show that $F_{\mu}\left(\partial V_{\mu}\right) \cap P_{n, \mu}=\emptyset$. (Note, that $V_{\mu}$ is bounded.) $F_{\mu}$ can be extended to all $\mathbb{R}^{r}$ by the formula (3). The system (2) describes $V_{\mu}$ is an open semialgebraic set in $\mathbb{R}^{r}$. By the Second Main Structural Theorem (see [BR, p. 68]) each point $v \in \partial V_{\mu}$ satisfies the following condition:

$$
\left[\bigvee_{i=1}^{r}\left(\left(v_{i}=b\right) \vee\left(v_{i}=c\right)\right)\right] \vee\left[\bigvee_{i \neq j}\left(v_{i}=v_{j}\right)\right]
$$

But by the definition of $P_{n, \mu}$ we get that $F_{\mu}(v) \notin P_{n, \mu}$ because the polynomial $F_{\mu}(v)$ has the different multiplicity symbol.

So, $P_{n, \mu}$ is a smooth submanifoled of $P_{n}$. The claim is proved.

Remark 2.1. Since $V_{\mu}$ is a semialgebraic set and $F_{\mu}$ is a semialgebraic map we obtain (by Tarski-Seidenberg Theorem) that $P_{n, \mu}$ is also a semialgebraic set.

Remark 2.2. Since $F_{\mu}$ is an immersion then

$$
\operatorname{dim} P_{n, \mu}=\operatorname{dim} V_{\mu}=r=n-\left(\sum_{i=1}^{r}\left(\mu_{i}-1\right)+\mu_{b}+\mu_{c}\right) .
$$

So, we obtained a formula for codimension of $P_{n, \mu}$ for this special case. 
Proof of the lemma: Suppose that $\sum_{i=1}^{r} \mu_{i}+\mu_{b}+\mu_{c}=m<n$. For each polynomial $f \in P_{m, \mu}$ we have:

$$
f=g \cdot p
$$

where $g \in P_{m, \mu}$ and $p \in P_{n-m,\{0,0\}}$.

Let $\widetilde{F}_{\mu}: P_{m, \mu} \times P_{n-m,\{0,0\}} \rightarrow P_{n}$ be the map defined by the formula (8). The map is one-to-one because a polynomial is uniquely defined by it's roots and sets of roots of $g$ and $p$ do not intersect.

Let us prove that $\widetilde{F}_{\mu}$ is an immersion. The proof uses the same arguments as the proof of the claim. Consider the same set $V_{\mu}$ described by inequalities (2) and construct a map $G_{\mu}: V_{\mu} \times P_{n-m,\{0,0\}} \rightarrow P_{n}$ such that

$$
G_{\mu}(v, p)=F_{\mu}(v) \cdot p
$$

where $F_{\mu}$ is a map constructed in the proof of the claim (the formula (3)). Since $F_{\mu}: V_{\mu} \rightarrow P_{m, \mu}$ is a diffeomorphism it is enough to prove that $G_{\mu}$ is an immersion.

Let us prove it by induction by $m$. If $m=0$ then $G_{\mu}$ is just the identity map on $P_{n-m,\{0,0\}}$ and $P_{n-m,\{0,0\}}$ is an open set (by Lemma 2.1). Suppose that we proved the statement for $m<m_{0}$. Let's prove it for $m_{0}$. Consider the polynomial $f \in P_{n, \mu}$. Suppose that $r \neq 0$. Take the root $v_{r}$. We have the following presentation

$$
f=\left(u-v_{r}\right)^{\mu_{r}} \cdot h
$$

where $h \in P_{n-\mu_{r}, \nu}, \nu=\left\{\mu_{b}, \mu_{1}, \ldots, \mu_{r-1}, \mu_{c}\right\}$.

The continuation of the proof is the same as in the claim.

Let $r=0$. It means that $\mu=\left\{\mu_{b}, \mu_{c}\right\}$. In this case $V_{\mu}=\emptyset$. Thus it is enough to prove that the maps $G_{\mu_{b}}: P_{n-\mu_{b},\{0,0\}} \rightarrow P_{n}$ and $G_{\mu_{c}}$ : $P_{n-m,\{0,0\}} \rightarrow P_{n-\mu_{b}}$ defined as follows

$$
G_{\mu_{b}}(h)=(u-b)^{\mu_{b}} \cdot h, \quad G_{\mu_{c}}(g)=(u-c)^{\mu_{c}} \cdot g
$$

are immersions.

We will show it for $G_{\mu_{b}}$. For $G_{\mu_{c}}$ the proof is the same.

Let $h \in P_{n-\mu_{b},\{0,0\}}$. Putting $\tilde{u}=u-b$ we obtain

$$
h(\tilde{u})=\tilde{u}^{n-\mu_{b}}+h_{n-\mu_{b}-1} \tilde{u}^{n-\mu_{b}-1}+\cdots+h_{0}
$$


and

$$
G_{\mu_{b}}(h)(\tilde{u})=\tilde{u}^{n}+h_{n-\mu_{b}-1} \tilde{u}^{n-1}+\cdots+h_{0} \tilde{u}^{\mu_{b}} .
$$

So, $P_{n, \mu}=G_{\mu_{b}} \circ G_{\mu_{c}}\left(P_{n-m,\{0,0\}}\right)$ is an immersed submanifold.

Let us prove that $P_{n, \mu}$ is a submanifold. The map $G_{\mu}$, defined by formula (9), can be extended to the set $\mathbb{R}^{r} \times P_{n-m}$. Let $(v, p) \in \partial\left(V_{\mu} \times\right.$ $\left.P_{n-m,\{0,0\}}\right)$. It means that either $v \in \partial V_{\mu}$ or $p \in \partial P_{n-m,\{0,0\}}$. The case $v \in \partial V_{\mu}$ was considered in the proof of the claim. Let $p \in \partial P_{n-m,\{0,0\}}$. It means that $P$ has a root in $[b, c]$. Hence, $f=G_{\mu}(v, p)$ does not belong to $P_{n, \mu}$.

Since $P_{n-m,\{0,0\}}$ is unbounded it is also necessary to prove that if a sequence $\left(v^{k}, p^{k}\right)$ tends to infinity for $k \rightarrow \infty$ then so for $G_{\mu}\left(v^{k}, p^{k}\right)$. But it is a partial case of Proposition 1.5.5 [BR].

Since $G_{\mu}$ is a homeomorphism to the image

$$
\operatorname{dim} P_{n, \mu}=r+n-m=n-\left(\sum_{i=1}^{r}\left(\mu_{i}-1\right)+\mu_{b}+\mu_{c}\right) .
$$

It proves the codimension formula. Lemma 2.2 is proved.

Remark 2.3. We also proved that $P_{n, \mu}$ is a semialgebraic set.

Let's define a codimension of $\mu$ as a codimension of the corresponding stratum $P_{n, \mu}$ if $P_{n, \mu} \neq \emptyset: c(\mu)=\operatorname{codim} P_{n, \mu}$. This definition is correct (does not depend on $n$ ), because by Lemma 2.2 we have:

$$
c(\mu)=\sum_{i=1}^{r}\left(\mu_{i}-1\right)+\mu_{b}+\mu_{c}
$$

Now we are going to define some semiorder relation $\mathcal{R}$ on the set of multiplicity symbols $\mathcal{M}$. We do it in the following way.

1. If $c\left(\mu^{1}\right)=c\left(\mu^{2}\right)$ then $\left(\mu^{1}, \mu^{2}\right)$ and $\left(\mu^{2}, \mu^{1}\right)$ do not belong to $\mathcal{R}$.

2. Let $c\left(\mu^{2}\right)=c\left(\mu^{1}\right)+1$. Then $\left(\mu^{1}, \mu^{2}\right) \in \mathcal{R}$ if these symbols satisfy one of the following conditions:

a) $r^{2}=r^{1}+1, \mu_{b}^{1}=\mu_{b}^{2}, \mu_{c}^{1}=\mu_{c}^{2}$ and there exists $\mu_{j}^{2}$ such that $\mu_{i}^{2}=\mu_{i}^{1}$ for all $i \neq j$ (and hence $\mu_{j}^{2}=2$ ).

b) $r^{2}=r^{1}-1, \mu_{b}^{1}=\mu_{b}^{2}, \mu_{c}^{1}=\mu_{c}^{2}$ and there exist $\mu_{s}^{1}, \mu_{j}^{1}$ and $\mu_{k}^{2}$ such that $\mu_{i}^{2}=\mu_{i}^{1}$ for all $i \neq s, j, k$ (and hence $\mu_{s}^{1}+\mu_{j}^{1}=\mu_{k}^{2}$ ).

c) $r^{2}=r^{1}-1, \mu_{b}^{1}=\mu_{b}^{2}$ and there exists $\mu_{j}^{1}$ such that $\mu_{i}^{2}=\mu_{i}^{1}$ for all $i \neq j$ (and hence $\mu_{c}^{1}+\mu_{j}^{1}=\mu_{c}^{2}$ ). 
d) $r^{2}=r^{1}-1, \mu_{c}^{1}=\mu_{c}^{2}$ and there exists $\mu_{j}^{1}$ such that $\mu_{i}^{2}=\mu_{i}^{1}$ for all $i \neq j$ (and hence $\mu_{b}^{1}+\mu_{j}^{1}=\mu_{b}^{2}$ ).

e) $r^{2}=r^{1}, \mu_{i}^{1}=\mu_{i}^{2}$ for all $i$ and $\mu_{b}^{1}+1=\mu_{b}^{2}$.

f) $r^{2}=r^{1}, \mu_{i}^{1}=\mu_{i}^{2}$ for all $i$ and $\mu_{c}^{1}+1=\mu_{c}^{2}$.

(We denote by $r^{1}$ and $r^{2}$ the numbers of internal elements of $\mu^{1}$ and $\left.\mu^{2}\right)$.

We write $\mu \prec \nu$ if $(\mu, \nu) \in \mathcal{R}$.

3. Let $c(\mu)<c(\nu)$. Then $\mu \prec \nu$ if there exists a finite set of symbols $\mu=\mu^{1}, \mu^{2}, \ldots, \mu^{k}=\nu$ such that $\mu^{1} \prec \mu^{2} \prec \mu^{3} \prec \cdots \prec \mu^{k}=\nu$.

Proposition 2.1. $\mathcal{R}$ is a semiorder relation on $\mathcal{M}$.

The proof just follows from the definition of $\mathcal{R}$.

Proposition 2.2. If $\mu \prec \nu$ then $P_{n, \nu} \subset C \ell\left(P_{n, \mu}\right)$.

Proof: It is enough to prove this statement just in the case $c(\nu)=$ $c(\mu)+1$. So, we have to check all possibilities for pair $(\mu, \nu)(2 . \mathrm{a})-2 . \mathrm{e}))$.

2.a) Let $f \in P_{n, \nu}$. Then we have the presentation

$$
f(u)=\left(u-v_{j}\right)^{2} \cdot g(u),
$$

where $g \in P_{n-2, \mu}$ and $v_{j} \in(b, c)$ is not a root of $g$.

Consider a family of polynomials

$$
f_{\epsilon}(u)=\left[\left(u-v_{j}\right)^{2}+\epsilon\right] \cdot g(u) .
$$

If $\epsilon \rightarrow 0$ then $f_{\epsilon} \rightarrow f$, but $f_{\epsilon} \in P_{n, \mu}$ for $\epsilon>0$. It means that $f \in$ $C \ell\left(P_{n, \mu}\right)$.

2.b) Consider also $f \in P_{n, \nu}$. In this case we have:

$$
f(u)=\left(u-v_{k}\right)^{\nu_{k}} \cdot g(u),
$$

where $g \in P_{n-\nu_{k}, \bar{\nu}}$ and $v_{k}$ is not a root of $g$.

$\left(\bar{\nu}\right.$ is equal to $\nu$ without a coordinate $\nu_{k}$ :

$$
\left.\bar{\nu}=\left(\nu_{b}, \ldots, \nu_{k-1}, \nu_{k+1}, \ldots, \nu_{c}\right)\right) .
$$

Consider a family

$$
f_{\epsilon}(u)=\left(u-\left(v_{k}-\epsilon\right)\right)^{\mu_{s}} \cdot\left(u-\left(v_{k}+\epsilon\right)\right)^{\mu_{j}} \cdot g(u) .
$$


If $\epsilon \rightarrow 0$ then $f_{\epsilon} \rightarrow f$ and $f_{\epsilon} \in P_{n, \mu}$ for sufficiently small $\epsilon>0$.

2.c) For $f \in P_{n, \nu}$ we can consider the following presentation

$$
f(u)=(u-c)^{\mu_{j}} \cdot g(u),
$$

where $g \in P_{n-\mu_{j}, \bar{\mu}}$ ( $\bar{\mu}$ is equal to $\mu$ without a coordinate $\mu_{j}: \bar{\mu}=$ $\left.\left(\mu_{b}, \ldots, \mu_{j-1}, \mu_{j+1}, \ldots, \mu_{c}\right)\right)$.

Consider $f_{\epsilon}(u)=(u-(c-\epsilon))^{\mu_{j}} \cdot g(u)$.

If $\epsilon \rightarrow 0$ then $f_{\epsilon} \rightarrow f$ and $f_{\epsilon} \in P_{n, \mu}$ for sufficiently small $\epsilon>0$.

The case 2.d) can be considered by the same arguments.

2.e) Let $f \in P_{n, \nu}$. Consider the presentation

$$
f(u)=(u-b) \cdot g(u),
$$

where $g \in P_{n-1, \mu}$.

Let's define $f_{\epsilon}(u)=(u-(b-\epsilon)) \cdot g(u)$.

Clearly, $f_{\epsilon} \in P_{n, \mu}$ for $\epsilon>0$ and $f_{\epsilon} \rightarrow f$ if $\epsilon \rightarrow 0$.

The case 2.f) can be considered in the same manner.

Proposition 2.3. If $(\mu, \nu) \notin \mathcal{R}$ then $C \ell\left(P_{n, \mu}\right) \cap P_{n, \nu}=\emptyset$.

Proof: Consider the map $G_{\mu}: V_{\mu} \times P_{n-m,\{0,0\}} \rightarrow P_{n}$ defined in the proof of Lemma 2 (the formula (9)). This map can be extended to $\mathbb{R}^{r} \times P_{n-m}$. We obtain

$$
G: \mathbb{R}^{r} \times P_{n-m} \rightarrow P_{n} \quad \text { and }\left.\quad G\right|_{V_{\mu} \times P_{n-m,\{0,0\}}}=G_{\mu} .
$$

By $[\mathbf{B R}]$ we know that this map is proper. From the definition of the semiorder $\mathcal{R}$ we obtain that

$$
G\left(C \ell\left(V_{\mu} \times P_{n-m,\{0,0\}}\right)\right)=\left(\bigcup_{\eta \succ \mu} P_{n, \eta}\right) \cup P_{n, \mu} .
$$

Since $G$ is a proper map it is also a closed map. So

$$
P_{n, \mu} \cup\left(\bigcup_{\eta \succ \mu} P_{n, \eta}\right)
$$

is a closed set. Since $P_{n, \mu} \cap P_{n, \nu}=\emptyset$ and for each $\eta \succ \mu, P_{n, \eta} \cap P_{n, \nu}=\emptyset$. Hence, $C \ell\left(P_{n, \mu}\right) \cap P_{n, \nu}=\emptyset$.

So we verified all conditions for semialgebraic stratification. Theorem is proved. 
2.2. Finite set of segments. Some generalizations of the stratifications.

Here we present some other stratifications of $P_{n}$ different from the first one but connected to it.

Let $\left\{\left[b_{i}, c_{i}\right], i=1, \ldots, s\right\}$ be a finite set of segments such that $\left[b_{i}, c_{i}\right] \cap$ $\left[b_{j}, c_{j}\right]=\emptyset$ for $i \neq j$. We can define corresponding multiplicity symbols in the following way.

A. Let $\alpha=\left\{\alpha_{b_{1}}, \ldots, \alpha_{b_{s}}, \alpha_{1}, \ldots, \alpha_{r}, \alpha_{c_{1}}, \ldots, \alpha_{c_{s}}\right\}$, where $r, \alpha_{b_{i}}, \alpha_{c_{i}}$ and $\alpha_{j}$ are integer numbers satisfying the following conditions:

1) $r \geq 0$

2) $\alpha_{b_{i}} \geq 0, \alpha_{c_{i}} \geq 0$,

3) $\alpha_{j}>0$ for $j=1, \ldots, r$ if $r \neq 0$,

4) $\alpha_{1} \leq \alpha_{2} \leq \cdots \leq \alpha_{r}$.

B. Let $\beta=\left\{\beta_{B}, \beta_{1}, \ldots, \beta_{r}\right\}$ be a set of integer numbers satisfying the following conditions:

1) $r \geq 0$

2) $\beta_{B} \geq 0$

3) $\beta_{j}>0$ for $j=1, \ldots, r$ if $r \neq 0$,

4) $\beta_{1} \leq \beta_{2} \leq \cdots \leq \beta_{r}$.

Let $\mathcal{A}$ be a set of multiplicity symbols defined in $A$ and $\mathcal{B}$ be a set of multiplicity symbols defined in $B$. Let $\alpha \in \mathcal{A}$. Define $P_{n, \alpha}$ as a set of polynomials $f$ from $P_{n}$ satisfying the following conditions:

1. At the boundary points $b_{i}$ and $c_{i}, f$ has roots of multiplicities $\alpha_{b_{i}}$ and $\alpha_{c_{i}}$ correspondently.

2. $f$ has exactly $r$ different roots $v_{1}, \ldots, v_{r}$ on the open set $\bigcup_{i=1}^{s}\left(b_{i}, c_{i}\right)$ and at each point $v_{j} f$ has a root of multiplicity $\alpha_{j}$.

Let $\beta \in \mathcal{B}$. Define $P_{n, \beta}$ as a set of polynomials $f$ from $P_{n}$ satisfying the following conditions:

$1^{*}$. The sum of all multiplicities of all roots of $f$ at all boundary points $b_{i}, c_{i}$ is equal to $\beta_{B}$

$2^{*}$. is the same as 2 .

Remark 2.4. If $s=1$ the stratification $\left\{P_{n, \alpha}\right\}_{\alpha \in \mathcal{A}}$ is the same as the stratification $\left\{P_{n, \mu}\right\}_{\mu \in \mathcal{M}}$ defined in section 2.1. 
Theorem 2.2. For each finite set of segments $\left\{\left[b_{i}, c_{i}\right], i=1, \ldots, s\right\}$ $\left\{P_{n, \alpha}\right\}_{\alpha \in \mathcal{A}}$ and $\left\{P_{n, \beta}\right\}_{\beta \in \mathcal{B}}$ are semialgebraic stratifications of $P_{n}$.

The proof is the same as the proof of Theorem 2.1.

\subsection{Interval Exchange Transformations.}

Let us recall a definition of Interval Exchange Transformation $[\mathbf{K e}]$. Consider some semiopen interval $[s, t) \subset \mathbb{R}$. Let $\left\{s_{i}\right\}(i=0, \ldots, m)$ such that $s=s_{0}<s_{1}<\cdots<s_{m-1}<s_{m}=t$ be a finite sequence of numbers defining a finite partition of $[s, t)$ to semiopen intervals $\Delta_{i}=\left[s_{i}, s_{i+1}\right)$. Interval Exchange Transformation is a map $T:[s, t) \rightarrow[s, t)$ satisfying the following conditions:

1. $T$ is a bijection.

2. At each subinterval $\Delta_{i} T$ is a translation: $\left.T(u)\right|_{\Delta_{i}}=u+\alpha_{i}$ $\left(\alpha_{i} \in \mathbb{R}\right)$.

Set $T(u)=u$ for $u \notin[s, t)$.

Let $[b, c]$ be some fixed segment. Denote by $G T$ the set of all interval exchange transformations non trivially defined on all $[s, t) \subset[b, c]$ such that $s \neq b$.

\section{Proposition 2.4.}

1. GT is a group with composition as a group operation.

2. There exists a natural action of the group $G T$ on the spaces $P_{n}$ such that all the strata $P_{n, \mu}$ are invariant by this action.

Proof: 1. It follows from the definition of Interval Exchange Transforamtions: associativity we obtain from bijectivity, a unity is the identity map and the inverse map for each interval exchange transformation is also an interval exchange transformation.

2. Let $f$ be a polynomial of degree $n: f \in P_{n}$. Let $T$ be some interval exchange transformation defined on some semiopen interval $[s, t): T \in$ $G T$. Let $v_{1}, \ldots, v_{r}$ be roots of $f$ belonging to $[s, t)$. We have the following presentation

$$
f=\left(u-v_{1}\right)^{\mu_{1}} \cdot \ldots \cdot\left(u-v_{r}\right)^{\mu_{r}} \cdot g(u),
$$

where $g$ is a polynomial which has no roots on $[s, t)$. Set

$$
F_{T}(f)=\left(u-T\left(v_{1}\right)\right)^{\mu_{1}} \cdot \ldots \cdot\left(u-T\left(v_{r}\right)\right)^{\mu_{r}} \cdot g(u) .
$$

It is easy to see that a map $\mathcal{F}: G T \times P_{n} \rightarrow P_{n}$ defined by $\mathcal{F}(T, f)=$ $F_{T}(f)$ is a group action. All strata $P_{n, \mu}$ are invariant by this action (it follows from the definition of $\left.P_{n, \mu}\right)$. 
Now we are going to define another action of $G T$ on $P_{n}$ which can be useful for our problems. Consider a set $R_{\epsilon, T}=(-\epsilon, \epsilon) \times[s, t)$. Let $\widetilde{T}_{\epsilon}: \mathbb{C} \rightarrow \mathbb{C}$ be the following map:

$$
\widetilde{T}_{\epsilon}(z)= \begin{cases}T(x)+i y & \text { for } z=x+i y \in R_{\epsilon, T} \\ z & \text { for } z \notin R_{\epsilon, T} .\end{cases}
$$

Let $\mathcal{F}_{\epsilon}: G T \times P_{n} \rightarrow P_{n}$ be a map defined as follows: $\mathcal{F}_{\epsilon}(T, f)=F_{T, \epsilon}(f)$, where $F_{T, \epsilon}(f)=\left(u-\widetilde{T}_{\epsilon}\left(v_{1}\right)\right)^{\mu_{1}} \cdot \ldots \cdot\left(u-\widetilde{T}_{\epsilon}\left(v_{k}\right)\right)^{\mu_{k}}$ for $f=\left(u-v_{1}\right)^{\mu_{1}}$. $\ldots \cdot\left(u-v_{k}\right)^{\mu_{k}}$. Here $v_{1}, \ldots, v_{k}$ are all roots of $f$.

Proposition 2.5. $\mathcal{F}_{\epsilon}$ is a group action. Strata $P_{n, \mu}$ are invariant by this action.

It is clear that the map $F_{T, \epsilon}$ is not necessary continuous on $P_{n}$. But we can formulate some continuity property of this map, which will be useful for analysing the geometry of the strata $P_{n, \mu}$. We will use a notation $z(f)$ for the set of all roots of $f$.

Theorem 2.3. Let $f \in P_{n}$ be a polynomial of degree $n$. Let $T$ be an interval exchange transformation defined on $[s, t)$ such that $s>b, t<c$ and for each $v \in z(f) \cap[s, t)$ there exists a subinterval $\Delta_{i} \subset[s, t)$ such that $v \in \operatorname{Int} \Delta_{i}$.

Then there exist $\epsilon>0$ and a neighbourhood $U_{f}$ such that $\left.F_{T, \epsilon}\right|_{U_{f}}$ is a diffeomorphism to the image.

Proof:

Step 1: Let us consider the case that $v$ be a unique root of $f$ and the multiplicity of $v$ is equal to $n$. In other words, $f=(u-v)^{n}$. Let $\Delta_{i}$ be a subinterval such that $v \in \operatorname{Int} \Delta_{i}$. It means that for some $\delta>0$ $(v-\delta, v+\delta) \subset \Delta_{i}$. Let $U_{f}$ be a neighbourhood of $f$ and $\epsilon>0$ such that for each $g \in U_{f}$ we have $z(g) \subset(-\epsilon, \epsilon) \times(v-\delta, v+\delta)$. We have a presentation

$$
g=(u-v)^{n}+g_{n-1}(u-v)^{n-1}+\cdots+g_{0} .
$$

By definition of $\widetilde{T}_{\epsilon}$ we obtain

$$
F_{T, \epsilon}(g)=(u-T(v))^{n}+g_{n-1}(u-T(v))^{n-1}+\cdots+g_{0} .
$$

Obvious, this map $F_{T, \epsilon}$ is diffeomorphism. 
Step 2: Let $T$ be a transformation such that there exists a unique root $v$ of $f$ such that $T(v) \notin z(f)$ and for other roots $v^{\prime} \in[b, c]$ we have $T\left(v^{\prime}\right)=v^{\prime}$. Then we obtain

$$
f=(u-v)^{\mu_{v}} \cdot \tilde{h}(u)
$$

Set $\tilde{f}=(u-v)^{\mu_{v}}$. Now take $\delta>0, \epsilon>0$ a neighbourhood $U_{\tilde{f}} \subset P_{\mu_{v}}$, a neighbourhood $U_{\tilde{h}} \subset P_{n-\mu_{v}}$ such that for each $q \in U_{\tilde{f}}$ and for each $h \in$ $U_{\tilde{h}}$ we have: $z(q) \subset(-\epsilon, \epsilon) \times(v-\delta, v+\delta), z(h) \cap((-\epsilon, \epsilon) \times(v-\delta, v+\delta))=\emptyset$ and $T$ is continuous on $(v-\delta, v+\delta)$. Consider a neighbourhood $U_{f} \subset P_{n}$ defined as follows: $U_{f}=U_{\tilde{f}} \cdot U_{\tilde{h}}$. (The symbol $\cdot$ means a usual product of polynomials). Thus for each $g \in U_{f}$ we have

$$
\begin{aligned}
& g=\left((u-v)^{\mu_{v}}+q_{\mu_{v}-1}(u-v)^{\mu_{v}-1}+\cdots+q_{0}\right) \\
& \cdot\left(u^{n-\mu_{v}}+h_{n-\mu_{v}-1} u^{n-\mu_{v}-1}+\cdots+h_{0}\right) .
\end{aligned}
$$

So,

$$
\begin{aligned}
F_{T, \epsilon}(g)=\left((u-T(v))^{\mu_{v}}+\right. & \left.\cdots+q_{0}\right) \\
& \cdot\left(u^{n-\mu_{v}}+h_{n-\mu_{v}-1} u^{n-\mu_{v}-1}+\cdots+h_{0}\right) .
\end{aligned}
$$

We see that $\left.F_{T, \epsilon}\right|_{U_{\tilde{f}}}$ is a diffeomorphism, $\left.F_{T, \epsilon}\right|_{U_{\tilde{h}}}$ is the identity map. By the straightforward calculations we obtain that the Jacobian matrix for the product operation on $U_{\tilde{f}} \times U_{\tilde{h}}$ at the point $(\tilde{f}, \tilde{h})$ is the resultant of $\tilde{f}$ and $\tilde{h}[\mathbf{J}]$.

Analogically, the Jacobian matrix for the product operation on $U_{F_{T, \epsilon}(\tilde{f})} \times U_{\tilde{h}}$ at $\left(F_{T, \epsilon}(\tilde{f}), \tilde{h}\right)$ is the resultant of $F_{T, \epsilon}(\tilde{f})$ and $\tilde{h}$. Since the pair $(\tilde{f}, \tilde{h})$ (and correspondently $\left(F_{T, \epsilon}(\tilde{f}), \tilde{h}\right)$ ) has no common zeros on $\mathbb{C}$ these resultants are not equal to zero. So, the Jacobians are non degenerate and hence $\left.F_{T, \epsilon}\right|_{U_{f}}$ is a diffeomorphism.

Step 3: Let the pair $(T, f)$ satisfies the following conditions: there are two different roots $v^{1}$ and $v^{2}$ of $f$ such that $T\left(v^{1}\right)=v^{2}$ and $T\left(v^{2}\right)=v^{1}$ and for every other root $v \in[b, c]$ we have $T(v)=v$. In this case we obtain the following presentations: $f=(u-v)^{\mu_{v^{1}}}\left(u-v^{1}\right)^{\mu_{v^{2}}} \cdot \tilde{h}(u)$ and $F_{T, \epsilon}(f)=\left(u-v^{2}\right)^{\mu_{v^{1}}}\left(u-v^{1}\right)^{\mu_{v^{2}}} \cdot \tilde{h}(u)$. Set $f^{1}=\left(u-v^{1}\right)^{\mu_{v^{1}}}$, $f^{2}=\left(u-v^{2}\right)^{\mu_{v^{2}}}$. Take $\delta>0, \epsilon>0$ and neighbourhoods $U_{f^{1}}, U_{f^{2}}$ and $U_{\tilde{h}}$ such that for each $q^{1} \in U_{f^{1}}, q^{2} \in U_{f^{2}}$ and $h \in U_{\tilde{h}}$ we have:

1. $z\left(q^{1}\right) \subset(-\epsilon, \epsilon) \times\left(v^{1}-\delta, v^{1}+\delta\right), z\left(q^{2}\right) \subset(-\epsilon, \epsilon) \times\left(v^{2}-\delta, v^{2}+\delta\right)$.

2. $\left(v^{2}-\delta, v^{2}+\delta\right) \cap\left(v^{1}-\delta, v^{1}+\delta\right)=\emptyset$.

3. $z(h) \cap\left((-\epsilon, \epsilon) \times\left(\left(v^{2}-\delta, v^{2}+\delta\right) \cup\left(v^{1}-\delta, v^{1}+\delta\right)\right)\right)=\emptyset$.

4. $T$ is continuous on $\left(v^{1}-\delta, v^{1}+\delta\right)$ and $\left(v^{2}-\delta, v^{2}+\delta\right)$. 
Then applying the same arguments as in the Step 2 we obtain that $F_{T, \epsilon}$ is a diffeomorphism to the image in a neighbourhood of $f$.

Step 4:

Lemma 2.3. Let the pair $(T, f)$ satisfies the condition of the theorem.

Then there exist $\delta>0$, a finite set of points $\left\{w_{k}\right\}$ and a special interval exchange transformation $S:[s, t) \rightarrow[s, t)$ such that

1) There exist $\epsilon>0$ and a neighbourhood $U_{f}$ such that $\left.F_{S, \epsilon}\right|_{U_{f}}=$ $\left.F_{T, \epsilon}\right|_{U_{f}}$.

2) $\left[w_{k}-\delta, w_{k}+\delta\right] \cap\left[w_{j}-\delta, w_{j}+\delta\right]=\emptyset$ for $k \neq j$.

3) Let $\Omega=\bigcup_{k}\left[w_{k}-\delta, w_{k}+\delta\right)$. Then $\left.S\right|_{\Omega}$ is a permutation of intervals $\left\{\left[w_{k}-\delta, w_{k}+\delta\right)\right\}$ and $\left.S\right|_{[s, t)-\Omega}=\mathrm{id}$.

Proof: Set

$$
\left\{w_{k}\right\}=(z(f) \cap[s, t)) \cup T(z(f) \cap[s, t)) .
$$

Take $\delta>0$ such that it satisfies the property 2) and for every $v \in$ $z(f) \cap[s, t)$ we have $[v-\delta, v+\delta] \subset \operatorname{Int} \Delta_{i}\left(\Delta_{i}\right.$ is the corresponding to $v$ subinterval (see the condition of the theorem)).

Set

$$
\Omega^{\prime}=\bigcup_{v \in z(f) \cap[s, t)}[v-\delta, v+\delta) \text { and }\left.\quad S\right|_{\Omega^{\prime}}=\left.T\right|_{\Omega^{\prime}} .
$$

Let us extend the map $S$ to $\Omega-\Omega^{\prime}$ such that $\left.S\right|_{\Omega}$ will be a permutation of intervals.

And finally, let us extend $S$ to $[s, t)-\Omega$ as the identity map. A neighbourhood $U_{f}$ of $f$ and $\epsilon>0$ we can find in the same way as in the Steps 2 and 3.

Step 5: Now we can restrict our consideration to permutations of intervals. For each permutation $S$ of intervals we have: $S=S^{1} \circ S^{2} \circ$ $\cdots \circ S^{m}$, where $\left\{S^{i}\right\}_{i=1}^{m}$ are permutations of intervals corresponding to the standard generators of the permutation group $S_{p}\left(p=\#\left\{\omega_{i}\right\}\right)$. Each generator is an interchanging of a pair of intervals, which were considered in the Steps 2 and 3. It completes the proof of the theorem. 
2.4. Differential triviality of the stratifications defined by the multiplicity symbol.

Let $A$ be a stratified set and $\left\{K_{i}\right\}$ be a stratification. We say that this stratification is differentially trivial if for each stratum $K_{i}$ and for each two points $x_{1}, x_{2} \in K_{i}$ there exist neighbourhoods of these points $U_{x_{1}} \subset A$ and $U_{x_{2}} \subset A$ and a diffeomorphism $F: U_{x_{1}} \rightarrow U_{x_{2}}$ such that $F\left(U_{x_{1}} \cap K_{j}\right)=U_{x_{2}} \cap K_{j}$ for every $j$ such that $K_{i} \subset C \ell\left(K_{j}\right)$.

Theorem 2.4. The stratification $\left\{P_{n, \mu}\right\}_{\mu \in \mathcal{M}}$ is differentially trivial.

Proof: Let $f_{1}, f_{2} \in P_{n, \mu}$. Let $m=\sum_{i=1}^{r} \mu_{i}+\mu_{b}+\mu_{c}$. Then we have: $f_{1}=\tilde{f}_{1} \cdot g_{1}, \tilde{f}_{2} \cdot g_{2}$, where $\tilde{f}_{1}, \tilde{f}_{2} \in P_{m, \mu}$ and $g_{1}, g_{2} \in P_{n-m,\{0,0\}}$.

Lemma 2.4. There exist neighbourhoods $U_{\tilde{f}_{1}}$ of $\tilde{f}_{1}$ and $U_{\tilde{f}_{2}}$ of $\tilde{f}_{2}$ in $P_{m}$ and a diffeomorphism $H: U_{\tilde{f}_{1}} \rightarrow U_{\tilde{f}_{2}}$ such that for each $\nu \prec \mu$ $H\left(P_{m, \nu} \cap U_{\tilde{f}_{1}}\right)=P_{m, \nu} \cap U_{\tilde{f}_{2}}$ and $H\left(P_{m, \mu} \cap U_{\tilde{f}_{1}}\right)=P_{m, \mu} \cap U_{\tilde{f}_{2}}$.

Proof of the lemma: Let $\mu=\left\{\mu_{b}, \mu_{1}, \ldots, \mu_{r}, \mu_{c}\right\}, v_{i} \in(b, c)$ and $\omega_{i} \in$ $(b, c)(i=1, \ldots, r)$ be roots of $\tilde{f}_{1}$ and $\tilde{f}_{2}$ (correspondently) corresponding to $\mu_{i}$. Take $\delta>0$ such that for all $i=1, \ldots, r$ we have:

1) $\left[v_{i}-\delta, v_{i}+\delta\right] \cap\left[v_{j}-\delta, v_{j}+\delta\right]=\emptyset$ if $i \neq j$.

2) $\left[v_{i}-\delta, v_{i}+\delta\right] \cap[b, b+\delta]=\emptyset$.

3) $\left[v_{i}-\delta, v_{i}+\delta\right] \cap[c-\delta, c]=\emptyset$.

Let for this $\delta$ the same properties hold for $\omega_{i}$ and

4) $\left[v_{i}-\delta, v_{i}+\delta\right] \cap\left[w_{j}-\delta, w_{j}+\delta\right]=\emptyset$ if $w_{j} \neq v_{i}$.

Denote by $\Omega_{1}=\bigcup_{i=1}^{r}\left[v_{i}-\delta, v_{i}+\delta\right), \Omega_{2}=\bigcup_{i=1}^{r}\left[w_{i}-\delta, w_{i}+\delta\right)$ and $\Omega=$ $\Omega_{1} \cup \Omega_{2}$. Let us define a permutation $S$ of intervals on $[b, c]$ in the following way.

Step 1: Define $S$ on $\Omega_{1}: S(u)=u-v_{i}+w_{i}$ for $u \in\left[v_{i}-\delta, v_{i}+\delta\right)$.

Step 2: Define $S$ on $\Omega_{2}-\Omega_{1}$ such that $\left.S\right|_{\Omega}$ be a permutation of intervals.

Step 3: Define $S(u)=u$ for $u \in[b, c]-\Omega$.

It is clear that $F_{S, \epsilon}\left(\tilde{f}_{1}\right)=\tilde{f}_{2}$ for every $\epsilon>0$. By Proposition 2.5 we have $F_{S, \epsilon}\left(P_{m, \nu}\right)=P_{m, \nu}$ for every multiplicity symbol $\nu$. By Theorem 2.3 there exist neighbourhoods $U_{\tilde{f}_{1}}$ and $U_{\tilde{f}_{2}}$ such that $H=F_{S, \epsilon}$ is a diffeomorphism of $U_{\tilde{f}_{1}}$ onto $U_{\tilde{f}_{2}}$. The lemma is proved. 
Remark 2.5. The neighbourhoods $U_{\tilde{f}_{1}}$ and $U_{\tilde{f}_{2}}$ can be choosen such that for each $h \in U_{\tilde{f}_{1}} \cup U_{\tilde{f}_{2}}$ for sufficiently small $\delta^{\prime}>0, z(h) \subset(-\epsilon, \epsilon) \times$ $\left(b-\delta^{\prime}, c+\delta^{\prime}\right)$.

Now take $\epsilon, \delta^{\prime}$ and neighbourhoods $U_{g_{1}}$ and $U_{g_{2}}$ such that:

1. For each $g \in U_{g_{1}} \cup U_{g_{2}} z(g) \cap\left((-\epsilon, \epsilon) \times\left(b-\delta^{\prime}, c+\delta^{\prime}\right)\right)=\emptyset$.

2. The map $L(g)=g-g_{1}+g_{2}$ maps $U_{g_{1}}$ onto $U_{g_{2}}$.

Let $U_{f_{1}}=U_{\tilde{f}_{1}} \cdot U_{g_{1}}$ and $U_{f_{2}}=U_{\tilde{f}_{2}} \cdot U_{g_{2}}$. Define a map $\psi: U_{f_{1}} \rightarrow U_{f_{2}}$ in the following way. For each $p \in U_{f_{1}}$ we have a unique presentation $p=\tilde{p} \cdot g$ such that $\tilde{p} \in U_{\tilde{f}_{1}}, g \in U_{g_{1}}$ (because the resultant of $\tilde{f}_{1}$ and $g_{1}$ is not equal to zero). Set $\psi(p)=H(\tilde{p}) \cdot L(g)$. Since $H$ and $L$ are local diffeomorphisms and the resultant of $\tilde{f}_{2}$ and $g_{2}$ is nondegenerate $\psi$ is a diffeomorphism. By lemma it has required properties. Theorem 2.4 is proved.

Since the stratification is differentially trivial and semialgebraic it is Whitney regular (see, for example, [GWPL]). Hence we have

Theorem 2.5. The stratification $\left\{P_{n, \mu}\right\}_{\mu \in \mathcal{M}}$ defined in section 1 is Whitney regular (satisfies the axioms "a" and "b" of Whitney).

Theorem 2.6. The stratifications defined in section 2.2 are Whitney regular.

The proof is the same as the proof of Theorem 2.4.

\section{Stratifications of complex monic polynomials}

\subsection{Multiplicity symbol.}

Let $P_{n}(\mathbb{C})$ be a space of all monic polynomials of degree $n$ with complex coefficients. Let $N \subset \mathbb{C}$ be a semialgebraic closed subset. We have $N=\operatorname{Int}(N) \cup \operatorname{Smooth}(\partial N) \cup \operatorname{Sing}(\partial N)$, where $\operatorname{Smooth}(\partial N)$ is a set of $C^{\rho}$-smooth points of $\partial N$ (we can suppose the order of differentiability $0 \leq \rho \leq \infty), \operatorname{Sing}(\partial N) \stackrel{\text { def }}{=} \partial N-\operatorname{Smooth}(\partial N)$. Since $N$ is semialgebraic we have \# $\operatorname{Sing}(\partial N)<\infty$ for any $\rho$. The set $\operatorname{Smooth}(\partial N)$ can be obtained as a union of two connected components $S_{1}$ and $S_{2}$ defined in the following way: $S_{1}=\{x \in \operatorname{Smooth}(\partial N)$ and there exists $\epsilon>0$ such that $B(x, \epsilon) \cap \operatorname{Int}(N) \neq \emptyset\}, S_{2}=\operatorname{Smooth}(\partial N)-S_{1}(B(x, \epsilon)$ means a ball with the center $x$ and radius $\epsilon)$.

Now let us define a multiplicity symbol $\mu(N)=\left\{\mu_{1}, \ldots, \mu_{r_{1}}, \eta_{1}, \ldots, \eta_{r_{2}}\right.$, $\left.\zeta_{1}, \ldots, \zeta_{r_{3}}, \theta_{1}, \ldots, \theta_{r_{4}}\right\}$ as a collection of natural numbers corresponding to the set $N$ satisfy the following properties: 
1. $r_{1} \geq 0, r_{2} \geq 0, r_{3} \geq 0, r_{4}=\# \operatorname{Sing}(N)$.

2. If $\operatorname{Int}(N)=\emptyset$ then $r_{1}=r_{2}=0$.

3. If $S_{1}=\emptyset$ then $r_{2}=0$.

4. If $S_{2}=\emptyset$ then $r_{3}=0$.

5. $\sum_{i=1}^{r_{1}} \mu_{i}+\sum_{j=1}^{r_{2}} \eta_{j}+\sum_{s=1}^{r_{3}} \zeta_{s}+\sum_{k=1}^{r_{4}} \theta_{k} \leq n$.

6. $0<\mu_{1} \leq \mu_{2} \leq \cdots \leq \mu_{r_{1}}$,

$0<\eta_{1} \leq \eta_{2} \leq \cdots \leq \eta_{r_{2}}$

$0<\zeta_{1} \leq \zeta_{2} \leq \cdots \leq \zeta_{r_{3}}$.

Denote by $\mathcal{M}_{n}(N)$ the set of all multiplicity symbols for $N$ and $n$ fixed.

Let $P_{n, \mu(N)}(\mathbb{C})$ be the set of polynomials $f \in P_{n}(\mathbb{C})$ such that

1. $f$ has exactly $r_{1}$ roots $v_{1} \neq v_{2} \neq \cdots \neq v_{r_{1}}$ on $\operatorname{Int}(N)$ with multiplicities $\mu_{1}, \ldots, \mu_{r_{1}}$ (correspondently).

2. $f$ has exactly $r_{2}$ roots $\tilde{v}_{1} \neq \tilde{v}_{2} \neq \cdots \neq \tilde{v}_{r_{2}}$ on $S_{1}$ with multiplicities $\eta_{1}, \ldots, \eta_{r_{2}}$.

3. $f$ has exactly $r_{3}$ roots $v_{1}^{\prime} \neq v_{2}^{\prime} \neq \cdots \neq v_{r_{3}}^{\prime}$ on $S_{2}$ with multiplicities $\zeta_{1}, \ldots, \zeta_{r_{3}}$.

4. $f$ has a root at the point $y_{k} \in \operatorname{Sing}(N)$ with a multiplicity $\theta_{k}$. (In the case $f\left(y_{k}\right) \neq 0$ set $\left.\theta_{k}=0\right)$.

The main goal of this part is the following result.

Theorem 3.1. The collection $\left\{P_{n, \mu(N)}(\mathbb{C})\right\}_{\mu(N) \in \mathcal{M}_{n}(N)}$ is a $C^{\rho}$-differentially trivial (and thus Whitney regular) stratification of $P_{n}(\mathbb{C})$.

The proof contains several steps.

Definition 3.1. A zero-symbol $0(N)$ is a multiplicity symbol such that the first three parts $\mu, \eta$ and $\zeta$ are empty $\left(r_{1}=r_{2}=r_{3}=0\right)$ and all $\theta$-s are equal to zero.

Lemma 3.1. $P_{n, 0(N)}$ is an open semialgebraic subset of $P_{n}(\mathbb{C})$.

Proof: Each polynomial $f \in P_{n}(\mathbb{C})$ can be presented in the following form:

$$
f=\left(u-v_{1}\right)\left(u-v_{2}\right) \cdot \ldots \cdot\left(u-v_{n}\right),
$$

$v_{1}, v_{2}, \ldots, v_{n} \in \mathbb{C}$ are the roots of $f$. 
Let $v=\left(v_{1}, \ldots, v_{n}\right) \in \mathbb{C}^{n}$ be a "root vector". Consider a subset $V_{0(N)} \subset \mathbb{C}^{n}$ defined as follows

$$
V_{0(N)}=\left\{v \in \mathbb{C}^{n} ; v=\left(v_{1}, \ldots, v_{n}\right), v_{i} \notin N \text { for } i=1, \ldots, n\right\} .
$$

Let $Q: \mathbb{C}^{n} \rightarrow P_{n}(\mathbb{C})$ be a map obtained by opening brackets in (11). We have $P_{n, 0(N)}(\mathbb{C})=Q\left(V_{0(N)}\right)$. Thus $P_{n, 0(N)}(\mathbb{C})$ is semialgebraic (by Tarski-Seidenberg Theorem). Since $N$ is a closed subset of $\mathbb{C} P_{n, 0(N)}$ is open (by continuaty of roots $[\mathbf{B R}]$ ).

Lemma 3.2. For each $\mu(N) \in \mathcal{M}_{n}(N)$ the set $P_{n, \mu(N)}(\mathbb{C})$ is a semialgebraic subset and an immersed submanifold of $P_{n}(\mathbb{C})$.

To prove the lemma we need one proposition which is in fact the statement of the lemma in some particular case.

Proposition 3.1. Let $\mu(N)=\left\{\left(\mu_{i}\right),\left(\eta_{j}\right),\left(\zeta_{s}\right),\left(\theta_{k}\right)\right\} \in \mathcal{M}_{n}(N)$ and $\sum_{i=1}^{r_{1}} \mu_{i}+\sum_{j=1}^{r_{2}} \eta_{j}+\sum_{s=1}^{r_{3}} \zeta_{s}+\sum_{k=1}^{r_{4}} \theta_{k}=n$.

Then $P_{n, \mu(N)}(\mathbb{C})$ is a semialgebraic subset and an immersed submanifold of $P_{n}(\mathbb{C})$.

Proof: Let $V_{\mu(N)}=\left\{v=\left(v_{1}, \ldots, v_{r_{1}}, \tilde{v}_{1}, \ldots, \tilde{v}_{r_{2}}, \ldots, v_{1}^{\prime}, \ldots, v_{r_{3}}^{\prime}\right\}\right.$ be a subset of $\mathbb{C}^{r}\left(r=r_{1}+r_{2}+r_{3}\right)$ obtained as a set of solutions of the following system of equations and inequalities

$$
\left\{\begin{array}{l}
v_{i} \in \operatorname{Int}(N), \\
\tilde{v}_{j} \in S_{1} \\
v_{s}^{\prime} \in S_{2}, \\
v_{i_{1}} \neq v_{i_{2}}, \tilde{v}_{j_{1}} \neq \tilde{v}_{j_{2}}, v_{s_{1}}^{\prime}=v_{s_{2}}^{\prime} \quad \text { for } i_{1} \neq i_{2}, j_{2} \neq j_{2}, s_{1} \neq s_{2} .
\end{array}\right.
$$

By the definition $V_{\mu(N)}$ is a $C^{\rho}$ semialgebraic submanifold of $\mathbb{C}^{r}$.

Let $F_{\mu(N)}: \mathbb{C}^{r} \rightarrow P_{n}(\mathbb{C})$ be a map defined in the following way

$$
\begin{aligned}
& F_{\mu(N)}\left(v_{1}, \ldots, v_{r_{1}}, \tilde{v}_{1}, \ldots, \tilde{v}_{r_{2}}, v_{1}^{\prime}, \ldots, v_{r_{3}}^{\prime}\right) \\
& =\left(u-v_{1}\right)^{\mu_{1}} \cdot \ldots \cdot\left(u-v_{r_{1}}\right)^{\mu_{r_{1}}}\left(u-\tilde{v}_{1}\right)^{\eta_{1}} \cdot \ldots \cdot\left(u-\tilde{v}_{2}\right)^{\eta_{r_{2}}}\left(u-v_{1}^{\prime}\right)^{\zeta_{1}} \\
& \cdot \ldots \cdot\left(u-v_{r_{3}}^{\prime}\right)^{\zeta_{r_{3}}}\left(u-y_{1}\right)^{\theta_{1}} \cdot \ldots \cdot\left(u-y_{r_{4}}\right)^{\theta_{r_{4}}}
\end{aligned}
$$

where $\operatorname{Sing}(\partial N)=\left\{y_{1}, \ldots, y_{r_{4}}\right\}$. Observe that if $\theta_{k}=0$ we have $(u-$ $\left.y_{k}\right)^{\theta_{k}}=1$.

Clearly the map $F_{\mu(N)}$ is an algebraic map. The proof of the fact that $F_{\mu(N)}$ is a local immersion is the same as in Lemma 2.2. 
Proof of Lemma 3.2: Now consider a general case:

$$
\sum_{i=1}^{r_{1}} \mu_{i}+\sum_{j=1}^{r_{2}} \eta_{j}+\sum_{s=1}^{r_{3}} \zeta_{s}+\sum_{k=1}^{r_{4}} \theta_{k} \leq n
$$

Each polynomial $f \in P_{n, \mu(N)}(\mathbb{C})$ can be presented in the following form:

$$
f=g \cdot h
$$

where $g \in P_{m, \mu(N)}(\mathbb{C})$ such that $m=\sum_{i=1}^{r_{1}} \mu_{i}+\sum_{j=1}^{r_{2}} \eta_{j}+\sum_{s=1}^{r_{2}} \zeta_{s}+\sum_{k=1}^{r_{4}} \theta_{k}$ and $h \in P_{n-m, 0(N)}(\mathbb{C})$.

We define a map $G_{\mu(N)}: V_{\mu(N)} \times P_{n-m, 0(N)}(\mathbb{C}) \rightarrow P_{n, \mu(N)}(\mathbb{C})$ in the following way:

$$
G_{\mu(N)}(v, h)=F_{\mu(N)}(v) \cdot h .
$$

By the same argument as in Lemma 2.2 we obtain that $G_{\mu(N)}$ is a local immersion. By Tarski-Seidenberg Theorem $P_{n, \mu(N)}(\mathbb{C})$ is a semialgebraic set.

Remark 3.1. In contrast to the real case the maps $F_{\mu(N)}$ and $G_{\mu(N)}$ are not necessary one-to-one. One can show that they are covering maps.

Remark 3.2. By the immediate calculations we obtain

$$
\operatorname{Codim} P_{n, \mu(N)}=2 \sum_{i=1}^{r_{1}}\left(\mu_{i}-1\right)+\sum_{j=1}^{r_{2}}\left(2 \eta_{j}-1\right)+\sum_{s=1}^{r_{3}}\left(2 \zeta_{s}-1\right)+2 \sum_{k=1}^{r_{4}} \theta_{k}
$$

In the next sections we are going to prove the differential triviality of the partition $\left\{P_{n, \mu(N)}(\mathbb{C})\right\}$.

\subsection{Disk-exchange transformations.}

Let $N \subset \mathbb{C}$ be a closed semialgebraic subset. Let $\left\{x_{1}, \ldots, x_{p}\right\}$ be a finite subset of $\operatorname{Int}(N)$. Consider $\epsilon>0$ such that

1. $B\left(x_{i}, \epsilon\right) \subset \operatorname{Int}(N)$ for all $i=1, \ldots, p$.

2. $B\left(x_{i_{1}}, \epsilon\right) \cap B\left(x_{i_{2}}, \epsilon\right)=\emptyset$ for $i_{1} \neq i_{2}$.

Let $\left\{\tilde{x}_{1}, \ldots, \tilde{x}_{t_{1}}\right\},\left\{x_{1}^{\prime}, \ldots, x_{i_{2}}^{\prime}\right\}$ be finite subsets of $S_{1}$ and $S_{2}$ correspondently and $\delta_{1}, \delta_{2}$ be numbers such that

3. Each pair $\left(B\left(\tilde{x}_{j}, \delta_{1}, B\left(\tilde{x}_{j}, \delta_{1}\right) \cap \partial N\right)\right.$ is $C^{\rho}$-diffeomorphic to the pair $(B(0,1), B(0,1) \cap \mathbb{R})$. 
Each pair $\left(B\left(x_{s}^{\prime}, \delta_{2}\right), B\left(x_{s}^{\prime}, \delta_{2}\right) \cap \partial N\right)$ is $C^{\rho}$-diffeomorphic to the pair $(B(0,1), B(0,1) \cap \mathbb{R})$.

4. $B\left(\tilde{x}_{j}, \delta_{1}\right) \cap B\left(x_{s}^{\prime}, \delta_{2}\right)=\emptyset$ for all $j=1, \ldots, t_{1}, s=1, \ldots, t_{2}$.

5. $B\left(\tilde{x}_{j_{1}}, \delta_{1}\right) \cap B\left(\tilde{x}_{j_{2}}, \delta_{1}\right)=\emptyset$ for $j_{1} \neq j_{2}$.

$B\left(x_{s_{1}}^{\prime}, \delta_{2}\right) \cap B\left(x_{s_{2}}^{\prime}, \delta_{2}\right)=\emptyset$ for $s_{1} \neq s_{2}$.

6. $B\left(x_{i}, \epsilon\right) \cap B\left(\tilde{x}_{j}, \delta_{1}\right)=\emptyset$

$B\left(x_{i}, \epsilon\right) \cap B\left(x_{s}^{\prime}, \delta_{2}\right)=\emptyset$ for all $i=1, \ldots, p, j=1, \ldots, t_{1}, s=$ $1, \ldots, t_{2}$.

Let

$$
B=\left(\bigcup_{i=1}^{p} B\left(x_{i}, \epsilon\right)\right) \cup\left(\bigcup_{j=1}^{t_{1}} B\left(\tilde{x}_{j}, \delta_{1}\right)\right) \cup\left(\bigcup_{s=1}^{t_{2}} B\left(x_{s}^{\prime}, \delta_{2}\right)\right)
$$

Definition 3.2. A map $T: \mathbb{C} \rightarrow \mathbb{C}$ is called a disk-exchange transformation associated to $N$ if it satisfies the following conditions:

1. $T$ is a bijection.

2. $\left.T\right|_{\mathbb{C}-B}=\mathrm{id}$.

3. For each $i_{1}$ there exists $i_{2}$ such that $\left.T\right|_{B\left(x_{i_{2}}, \epsilon\right)}$ is a $C^{\rho}$-diffeomorphism of $B\left(x_{i_{1}}, \epsilon\right)$ onto $B\left(x_{i_{2}}, \epsilon\right)$ such that $T\left(x_{i_{1}}\right)=x_{i_{2}}$.

4. For each $j_{1}$ (or $s_{1}$ ) there exists $j_{2}$ (or $s_{2}$, correspondently), such that $\left.T\right|_{B\left(\tilde{x}_{j_{1}}, \delta_{1}\right)}$ (or $\left.\left.T\right|_{B\left(x_{s_{1}}^{\prime}, \delta_{2}\right)}\right)$ is a $C^{\rho}$-diffeomorphism onto $B\left(\tilde{x}_{j_{2}} \delta_{1}\right)\left(\right.$ or $\left.B\left(x_{s_{2}}^{\prime}, \delta_{2}\right)\right)$ such that

$$
\begin{aligned}
& T\left(\tilde{x}_{j_{1}}\right)=\tilde{x}_{j_{2}} \quad\left(\text { or } T\left(x_{s_{1}}^{\prime}\right)=x_{s_{2}}^{\prime}\right) \quad \text { and } \\
& T\left(B\left(\tilde{x}_{j_{1}}, \delta_{1}\right) \cap N\right)=B\left(\tilde{x}_{j_{2}}, \delta_{1}\right) \cap N \\
& \quad\left(\text { or } T\left(B\left(x_{s_{1}}^{\prime}, \delta_{2}\right) \cap N\right)=B\left(x_{s_{2}}^{\prime}, \delta_{2}\right) \cap N\right. \text { correspondently). }
\end{aligned}
$$
tion.

The points $x_{i}, \tilde{x}_{j}, x_{s}^{\prime}$ we call centers of the disk-exchange transforma-

Let us define a map $T_{*}: P_{n}(\mathbb{C}) \rightarrow P_{n}(\mathbb{C})$ associated to a given diskexchange transformation $T$. Let $f \in P_{n}(\mathbb{C})$ be presented in the following standard form:

$$
f=\left(u-v_{1}\right)^{\alpha_{1}} \cdot \ldots \cdot\left(u-v_{k}\right)^{\alpha_{k}} .
$$

Set $T_{*}(f)=\left(u-T\left(v_{1}\right)\right)^{\alpha_{1}} \cdot \ldots \cdot\left(u-T\left(v_{k}\right)\right)^{\alpha_{k}}$. 
Proposition 3.2. Let $f \in P_{n, \mu(N)}(\mathbb{C})$. Then for each disk-exchange transformation $T$ associated to $N$ we have $T_{*}(f) \in P_{n, \mu(N)}(\mathbb{C})$.

This proposition follows from the fact that $N, \partial N$ and $\mathbb{C}-N$ are invariant under the map $T$.

Remark 3.3. The disk-exchange transformations associated to $N$ do not form a group for arbitrary chosen $N$.

Now we prove the theorem analogous to the Theorem 2.3.

Theorem 3.2. Let $\mu(N) \in \mathcal{M}_{m}(N)$ and

$$
\sum_{i=1}^{r_{1}} \mu_{i}+\sum_{j=1}^{r_{2}} \eta_{j}+\sum_{s=1}^{r_{3}} \zeta_{s}+\sum_{k=1}^{r_{4}} \theta_{k}=m .
$$

Let $f_{1}, f_{2} \in P_{m, \mu(N)}(\mathbb{C})$.

Then

1) There exists a disk-exchange transformation $T: \mathbb{C} \rightarrow \mathbb{C}$ such that $T_{*}\left(f_{1}\right)=f_{2}$.

2) There exist neighbourhoods $U_{f_{1}}$ and $U_{f_{2}}$ in $P_{m}(\mathbb{C})$ such that $T_{*}$ : $U_{f_{1}} \rightarrow U_{f_{2}}$ is a $C^{\rho}$-diffeomorphism.

Proof: 1) Let $Z_{1}$ be a zero set of $f_{1}$ and $Z_{2}$ be a zero set of $f_{2}$. We have $Z_{q}=Z_{1}^{1} \cup Z_{q}^{2} \cup Z_{q}^{3} \cup Z_{q}^{4}(q=1$ or 2$)$, where

$Z_{q}^{1}=Z_{q} \cap \operatorname{Int}(N), \quad Z_{q}^{2}=Z_{q} \cap S_{1}, \quad Z_{q}^{3}=Z_{q} \cap S_{2}, \quad Z_{q}^{4}=Z_{q} \cap \operatorname{Sing}(\partial N)$.

Let

$$
Z^{1}=Z_{1}^{1} \cup Z_{2}^{1}, \quad Z^{2}=Z_{1}^{2} \cup Z_{2}^{2}, \quad Z^{3}=Z_{1}^{3} \cup Z_{2}^{3}, \quad Z^{4}=Z_{1}^{4} \cup Z_{2}^{4} .
$$

(Observe that by the definition of the multiplicity symbol we have $Z^{4}=$ $\left.Z_{1}^{4}=Z_{2}^{4}\right)$.

Let $Z=Z_{1} \cup Z_{2}$. Let $P: Z \rightarrow Z$ be a permutation of points satisfies the following conditions:

1. $\left.P\right|_{Z^{4}}=\mathrm{id}$.

2. $P\left(Z_{1}^{\ell}\right)=Z_{2}^{\ell}(\ell=1,2,3,4)$.

3. If $v_{i} \in \operatorname{Int}(N)$ is a root of $f_{1}$ with multiplicity $\mu_{i}$ then $P\left(v_{i}\right)$ is a root of $f_{2}$ with the same multiplicity $\mu_{i}$.

4. If $\tilde{v}_{j} \in S_{1}$ is a root of $f_{1}$ with multiplicity $\eta_{j}$ then $P\left(\tilde{v}_{j}\right)$ is a root of $f_{2}$ with multiplicity $\eta_{j}$.

5. If $v_{s}^{\prime} \in S_{2}$ is a root of $f_{1}$ with multiplicity $\zeta_{s}$ then $P\left(v_{s}^{\prime}\right)$ is a root of $f_{2}$ with multiplicity $\zeta_{s}$. 
Let $Z^{1}=\left\{x_{1}, \ldots, x_{p}\right\}, Z^{2}=\left\{\tilde{x}_{1}, \ldots, \tilde{x}_{t_{1}}\right\}, Z^{3}=\left\{x_{1}^{\prime}, \ldots, x_{r_{2}}^{\prime}\right\}$. Let $\epsilon$, $\delta_{1}$ and $\delta_{2}$ be numbers such that conditions 1-6 from the definition of the disk-exchange transformation are satisfied. We define a disk-exchange transformation $T: \mathbb{C} \rightarrow \mathbb{C}$ with centers $x_{i}, \tilde{x}_{j}$ and $x_{s}^{\prime}$ by $T\left(x_{i}\right)=P\left(x_{i}\right)$, $T\left(\tilde{x}_{j}\right)=P\left(\tilde{x}_{j}\right)$ and $T\left(x_{s}^{\prime}\right)=P\left(x_{s}^{\prime}\right)$.

Clearly $T_{*}\left(f_{1}\right)=f_{2}$.

2) Step 1: Let us consider some particular case. Let $v_{i_{0}}^{1}, v_{i_{0}}^{2} \in \operatorname{Int}(N)$ be roots of $f_{1}$ and $f_{2}$ (correspondently) with multiplicity $\mu_{i_{0}}$. Suppose that other roots of $f_{1}$ and $f_{2}$ are the same; $v_{i}^{1}=v_{i}^{2}\left(i \neq i_{0}\right), \tilde{v}_{j}^{1}=\tilde{v}_{j}^{2}$, $v_{s}^{\prime 1}=v_{s}^{\prime 2}$ (for all $\left.j, s\right)$. The disk-exchange transformation $T$ constructed in the part 1) is just an interchanging of the two small disks with centers at $v_{i_{0}}^{1}$ and $v_{i_{0}}^{2}$ and radius $\epsilon$. We have

$$
\begin{aligned}
& f_{1}=\left(u-v_{i_{0}}^{1}\right)^{\mu_{i_{0}} \cdot h} \\
& f_{2}=\left(u-v_{i_{0}}^{2}\right)^{\mu_{i_{0}} \cdot h,}
\end{aligned}
$$

where $h \in P_{m-\mu_{i_{0}}}(\mathbb{C})$.

Let $U_{0}^{1}$ be a small neighbourhood of $\left(u-v_{i_{0}}^{1}\right)^{\mu_{i_{0}}}$ in $P_{\mu_{i_{0}}}(\mathbb{C})$ and $U_{h}$ be a small neighbourhood of $h$ in $P_{m-\mu_{0}}(\mathbb{C})$ such that the map $\phi_{1}: U_{0}^{1} \times U_{h} \rightarrow$ $P_{m}(\mathbb{C})$ obtained by "opening brackets" in (12) is a diffeomorphism to the image. ( $\phi_{1}$ is a local diffeomorphism because the Jacobian $J \phi_{1}$ at the point $\left(\left(u-v_{i_{0}}^{1}\right)^{\mu_{i}}, h\right)$ is a resultant of these polynomials $[\mathbf{J}]$ and they have not common roots). Analogically let $U_{0}^{2}$ be a small neighbourhood of $\left(u-v_{i_{0}}^{2}\right)^{\mu_{i_{0}}}$ in $P_{\mu_{0}}(\mathbb{C})$ such that $\phi_{2}: U_{0}^{2} \times U_{h} \rightarrow P_{m}(\mathbb{C})$ obtained by "opening brackets" in (13) is a diffeomorphism to the image. Let $U_{f_{1}}=\phi_{1}\left(U_{0}^{1} \times U_{h}\right)$ and $U_{f_{2}}=\phi_{2}\left(U_{0}^{2} \times U_{h}\right)$. Let $\widetilde{T}: U_{0}^{1} \rightarrow U_{0}^{2}$ be the map defined in the same way as $T_{*}$ but in the space $P_{\mu_{0}}(\mathbb{C})$. Observe that $\widetilde{T}$ is a diffeomorphism. Thus in the coordinate system defined by $\phi_{1}$ and $\phi_{2}$ the map $T_{*}$ can be written in the following way: $T_{*}=(\widetilde{T}$, id $)$. Since $\widetilde{T}$ is a diffeomorphism $T_{*}$ is also diffeomorphism.

Step 2: Let $\tilde{v}_{j_{0}}^{1}, \tilde{v}_{j_{0}}^{2}$ be roots of $f_{1}$ and $f_{2}$ (correspondently) with multiplicity $\eta_{j_{0}}$. Suppose also (as in Step 1) that other roots of $f_{1}$ and $f_{2}$ are the same. The proof is absolutely the same as in Step 1.

Step 3: The same arguments for $f_{1}$ and $f_{2}$ with roots $v_{s_{0}}^{\prime 1}, v_{s_{0}}^{\prime 2}$ with multiplicity $\zeta_{s_{0}}$ and the same other roots.

Step 4: For every pair $f_{1}, f_{2} \in P_{m, \mu(N)}(\mathbb{C})$ a disk-exchange transformation $T$ such that $T_{*}(1)=f_{2}$ can be obtained as a composition of maps considered in Steps 1, 2, 3.

The theorem is proved. 


\subsection{Differential triviality.}

Theorem 3.3. The family $\left\{P_{n, \mu(N)}(\mathbb{C})\right\}_{\mu(N) \in \mathcal{M}_{n}(N)}$ is $C^{\rho}$-differentially trivial.

Proof: Let $f_{1}, f_{2} \in P_{n, \mu(N)}(\mathbb{C})$ we have:

$$
\begin{aligned}
& f_{1}=\tilde{f}_{1} \cdot g_{1}, \\
& f_{2}=\tilde{f}_{2} \cdot g_{2},
\end{aligned}
$$

where $\tilde{f}_{1}, \tilde{f}_{2} \in P_{m, \mu(N)}(\mathbb{C})$ with $m=\Sigma \mu_{i}+\Sigma \eta_{j}+\Sigma \zeta_{s}+\Sigma \theta_{k}, g_{1}, g_{2} \in$ $P_{n-m, 0(N)}(\mathbb{C})$.

Let $T$ be a disk-exchange transformation connecting $\tilde{f}_{1}$ and $\tilde{f}_{2}$ constructed in Theorem 3.2. Let $U_{\tilde{f}_{1}}$ and $U_{\tilde{f}_{2}}$ be small neighbourhoods

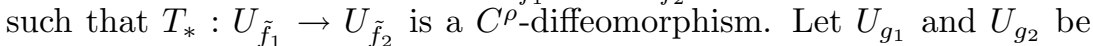
two small open balls in $P_{n-m}(\mathbb{C})$ belonging to $P_{n-m, 0(N)}(\mathbb{C})$. (These two balls exist because $P_{n-m, 0(N)}(\mathbb{C})$ is an open subset of $P_{n-m}(\mathbb{C})$ by Lemma 3.1). Let $H: U_{g_{1}} \rightarrow U_{g_{2}}$ be a diffeomorphism (can be a translation map). Let $\psi_{1}: U_{g_{1}} \times U_{\tilde{f}_{1}} \rightarrow P_{n}(\mathbb{C})$ and $\psi_{2}: U_{g_{2}} \times U_{\tilde{f}_{2}} \rightarrow P_{n}(\mathbb{C})$ be maps defined by "opening brackets" in (14) and (15). $\psi_{1}$ and $\psi_{2}$ are local diffeomorphisms by the same argument as in Theorem 3.2. Set $U_{f_{1}}=\psi_{1}\left(U_{g_{1}} \times U_{\tilde{f}_{1}}\right)$ and $U_{f_{2}}=\psi_{2}\left(U_{g_{2}} \times U_{\tilde{f}_{2}}\right)$. Thus the map

$$
G=\psi_{2} \circ\left(H, T_{*}\right) \circ \psi_{1}^{-1}: U_{f_{1}} \rightarrow U_{f_{2}}
$$

defines a required diffeomorphism.

End of the proof of Theorem 3.1: So, we obtained that the family $\left\{P_{n, \mu(N)}(\mathbb{C})\right\}_{\mu(N) \in \mathcal{M}_{n}(N)}$ satisfies the following conditions:

$$
\text { 1. } \bigcup_{\mu(N) \in \mathcal{M}_{n}(n)} P_{n, \mu(N)}(\mathbb{C})=P_{n}(\mathbb{C}) \text {. }
$$

2. $P_{n, \mu(N)}(\mathbb{C})$ are immersed submanifolds and semialgebraic subsets of $P_{n}(\mathbb{C})$.

3. $P_{n, \mu^{1}(N)}(\mathbb{C}) \cap P_{n, \mu^{2}(N)}(\mathbb{C})=\emptyset$ for $\mu^{1}(N) \neq \mu^{2}(N)$.

4. The family is $C^{\rho}$-differentially trivial.

Thus (by $\left[\right.$ GPWL]) we can conclude that $\left\{P_{n, \mu(N)}(\mathbb{C})\right\}_{\mu(N) \in \mathcal{M}_{n}(N)}$ is a Whitney regular stratification of $P_{n}(\mathbb{C})$. The theorem is proved. 


\section{Comparing of the stratifications}

\subsection{Comparing of real and complex cases.}

Now consider $N=[a, b] \subset \mathbb{C}$. Thus we have: $\operatorname{Int}(N)=\emptyset, S_{1}=\emptyset$, $S_{2}=(a, b)$ and $\operatorname{Sing}(\partial N)=\{a, b\}$. It means that the multiplicity symbol $\mu^{\mathbf{C}}([a, b])$ defined in the part 3 (complex case) has the following form:

$$
\mu^{\mathbf{C}}([a, b])=\left\{\zeta_{1}, \ldots, \zeta_{r_{3}}, \theta_{1}, \theta_{2}\right\} \quad\left(\text { here } r_{1}=r_{2}=0\right) .
$$

From the other side the multiplicity symbol $\mu^{\mathbf{R}}([a, b])$ corresponding to $[a, b]$ defined in the part 2 (real case) has the form: $\mu^{\mathbf{R}}([a, b])=$ $\left\{\mu_{a}, \mu_{1}, \ldots, \mu_{r}, \mu_{b}\right\}$.

Denote by $P_{n, \mu^{\mathbf{R}}([a, b])}(\mathbb{R})$ the stratum corresponding to the multiplicity symbol $\mu^{\mathbf{R}}([a, b])$ in real case, $P_{n, \mu} \mathbf{C}_{(N)}(\mathbb{C})$ the stratum corresponding to $\mu^{\mathbf{C}}([a, b])$ in complex case. Let $\mathcal{M}_{n}^{\mathbf{R}}([a, b])$ be a set of all multiplicity symbols $\mu^{\mathbf{R}}([a, b])$ and $\mathcal{M}_{n}^{\mathbf{C}}([a, b])$ be a set of all multiplicity symbols $\mu^{\mathbf{C}}([a, b])$.

Define a map $L: \mathcal{M}_{n}^{\mathbf{R}}([a, b]) \rightarrow \mathcal{M}_{n}^{\mathbf{C}}([a, b])$ by $L\left(\left\{\mu_{a}, \mu_{1}, \ldots, \mu_{r}, \mu_{b}\right\}\right)=$ $\left\{\mu_{1}, \ldots, \mu_{r}, \mu_{a}, \mu_{b}\right\}$. Clearly $L$ is a bijection. We have that $P_{n}(\mathbb{R})$ is a subset of $P_{n}(\mathbb{C})$.

Theorem 4.1. $P_{n, \mu^{\mathrm{R}}([a, b])}(\mathbb{R})=P_{n, L\left(\mu^{\mathrm{R}}([a, b])\right)}(\mathbb{C}) \cap P_{n}(\mathbb{R})$. This statement follows directly from the definitions of stratifications.

The same result is true for a finite number of intervals.

\subsection{Comparing of two complex stratifications.}

Let $\left\{X_{i}\right\}$ and $\left\{Y_{j}\right\}$ be two stratifications of $\mathbb{R}^{n}$. We say that these stratifications are $C^{\rho}$-equivalent if there exists a $C^{\rho}$-diffeomorphism $H: \mathbb{R}^{n} \rightarrow \mathbb{R}^{n}$ such that for each $i$ there exists $j$ such that $H\left(X_{i}\right)=Y_{j}$.

Remark 4.1. It is clear that if $\left\{X_{i}\right\}$ is $C^{\rho}$-equivalent to $\left\{Y_{j}\right\}$ that these stratifications has the same number of stratum and for each $i$ the sets $X_{i}$ and $Y_{j}=H\left(X_{i}\right)$ are $C^{\rho}$-diffeomorphic.

Let $N_{1}$ and $N_{2}$ are two semialgebraic subsets of $\mathbb{C}$. We say that $N_{1}$ and $N_{2}$ are $C^{\rho}$-equivalent if there exists a $C^{\rho}$-diffeomorphism $L: \mathbb{C} \rightarrow \mathbb{C}$ such that $L\left(N_{1}\right)=N_{2}$.

Let $f=\left(u-v_{1}\right)^{m_{1}}\left(u-v_{2}\right)^{m_{2}} \cdot \ldots \cdot\left(u-v_{k}\right)^{m_{k}} \in P_{n}(\mathbb{C})$. Define $L_{*}(f)$ as the following:

$$
L_{*}(f)=\left(u-L\left(v_{1}\right)\right)^{m_{1}}\left(u-L\left(v_{2}\right)\right)^{m_{2}} \cdot \ldots \cdot\left(u-L\left(v_{k}\right)\right)^{m_{k}} .
$$

We obtain a map $L_{*}: P_{n}(\mathbb{C}) \rightarrow P_{n}(\mathbb{C})$. 


\section{Theorem 4.2.}

1) $L_{*}$ is a $C^{\rho}$-diffeomorphism.

2) The stratifications

$$
\left\{P_{n, \mu\left(N_{1}\right)}(\mathbb{C})\right\}_{\mu\left(N_{1}\right) \in \mathcal{M}_{n}\left(N_{1}\right)} \text { and }\left\{P_{n, \mu\left(N_{2}\right)}(\mathbb{C})\right\}_{\mu\left(N_{2}\right) \in \mathcal{M}_{n}\left(N_{2}\right)}
$$

are $C^{\rho}$-equivalent.

3) The equivalence of these stratifications is given by $L_{*}$.

Proof: 1) This part follows from the properties of $L_{*}: 1 . L_{*}$ is a local diffeomorphism (the proof is the same as in Lemma 2.2). 2. $L_{*}$ is a one-to-one map. 3. $L_{*}$ is a proper map (see $[\mathbf{B R}]$ ).

The proof of 2) and 3) is the same as the proof of Theorem 3.1 of the part 3.

Remark 4.2. If $N_{1}$ and $N_{2}$ have a different number of $C^{\rho}$-singular points then the stratifications $\left\{P_{n, \mu\left(N_{1}\right)}(\mathbb{C})\right\}$ and $\left\{P_{n, \mu\left(N_{2}\right)}(\mathbb{C})\right\}$ have different number of strata and (by Remark 4.1) can not be $C^{\rho}$-equivalent. It means that different semialgebraic subsets define strictly different stratifications of $P_{n}(\mathbb{C})$.

Acknowledgments. The author was supported by CNPq grant $\mathrm{N}$ 300805/96-9.

I am grateful to the Department of Mathematics UFC and to Instituto de Matemática Pura e Aplicada (IMPA), Rio de Janeiro.

I would like to thank M. Peixoto who stimulated my interest to these problems and gave me many very useful suggestions.

I would like to thank A. Hefetz, M. Sobolevsky, K. Bekka, L. Jorge and Yu. Bilu for useful discussions and remarks.

I am grateful to $\mathrm{C}$. Gutierrez who gave me the idea that Interval Exchange Transformations can be used in Real Algebraic Geometry.

\section{References}

[AVG] V. Arnold, A. Varchenko and S. Gussein-Zade, "Singularities of differentiable maps," vol. 1, Birkhäuser, 1985.

[BCR] J. Bochnak, M. Coste and M.-F. Roy, "Géometrie algébrique réelle," Springer-Verlag, 1986.

[BR] R. Benedetti And J.-J. RisleR, "Real algebraic and semialgebraic sets," Hermann, 1990. 
[dPW] A. Du Plessis and C. T. C. Wall, "The Geometry of Topological Stability," Clarendon Press, Oxford, 1995.

[G] C. G. Gibson, Regularity of the Segre stratification, Math. Proc. Cambridge Philos. Soc. (1976), 80-91.

[GWPL] C. G. Gibson, K. Wirthmüller, A. A. DU Plessis And E. J. N. LoOIJENGA, "Topological stability of smooth mappings," Lecture Notes in Mathematics 552, Springer, 1976.

[J] N. JAcobson, "Lectures in abstract algebra," vol. 3, Van Nostrand, Princeton, 1964.

[Ke] M. Keane, Interval Exchange Transformations, Math. Z. 141 (1975), 25-31.

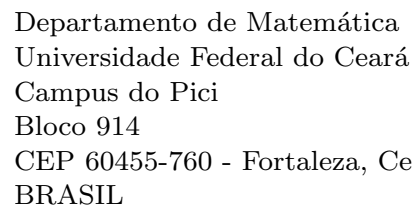

e-mail: lev@mat.ufc.br

Primera versió rebuda el 29 de maig de 1997, darrera versió rebuda el 30 d'octubre de 1997 